UICHEP-TH/97-6; IP/BBSR-97-23

\title{
Cyclic Shape Invariant Potentials
}

\section{Uday P. Sukhatme ${ }^{a, \text { 凹, Constantin Rasinariu }}{ }^{a, \text { f }}$ and Avinash Khare ${ }^{b, \beta}$}

a) Department of Physics (m/c 273), University of Illinois at Chicago, 845 W. Taylor Street, Chicago, Illinois 60607-7059,USA

b) Institute of Physics, Sachivalaya Marg, Bhubaneswar 751005, India

\begin{abstract}
We formulate and study the set of coupled nonlinear differential equations which define a series of shape invariant potentials which repeats after a cycle of $p$ iterations. These cyclic shape invariant potentials enlarge the limited reservoir of known analytically solvable quantum mechanical eigenvalue problems. At large values of $x$, cyclic superpotentials are found to have a linear harmonic oscillator behavior with superposed oscillations consisting of several systematically varying frequencies. At the origin, cyclic superpotentials vanish when the period $p$ is odd, but diverge for $p$ even. The eigenvalue spectrum consists of $p$ infinite sets of equally spaced energy levels, shifted with respect to each other by arbitrary energies $\omega_{0}, \omega_{1}, \ldots, \omega_{p-1}$. As a special application, the energy spacings $\omega_{k}$ can be identified with the periodic points generated by the logistic map $z_{k+1}=r z_{k}\left(1-z_{k}\right)$. Increasing the value of $r$ and following the bifurcation route to chaos corresponds to studying cyclic shape invariant potentials as the period $p$ takes values $1,2,4,8, \ldots$
\end{abstract}

\footnotetext{
${ }^{1}$ sukhatme@uic.edu

${ }^{2}$ costel@uic.edu

${ }^{3}$ khare@iop.ren.nic.in
} 
The number of analytically solvable eigenvalue problems in quantum mechanics is rather limited. In this regard, the concept of shape invariance [1] in supersymmetric quantum mechanics [2, [3] has proved to be very useful because it leads immediately to exactly solvable potentials. In this paper, we define and study cyclic shape invariant potentials. The supersymmetric partner Hamiltonians correspond to a series of shape invariant potentials which repeats after a cycle of $p$ iterations. Such potentials have an infinite number of periodically spaced eigenvalues. More precisely, the level spacings are given by $\omega_{0}, \omega_{1}, \ldots, \omega_{p-1}, \omega_{0}, \omega_{1}, \ldots, \omega_{p-1}, \omega_{0}, \omega_{1}, \ldots$, (see Fig. 1). The ground state is at zero energy; the next $(p-1)$ eigenvalues are $E_{l}=\sum_{k=0}^{l} \omega_{k}, l=0,1, \ldots,(p-2)$, and all other eigenvalues are obtained by adding arbitrary multiples of the quantity $\Omega_{p} \equiv \omega_{0}+\omega_{1}+\cdots+\omega_{p-1}$. The general formula for the excited energy levels is

$$
n \Omega_{p}+\sum_{k=0}^{l} \omega_{k} ;\{n=0,1,2, \ldots, \infty \quad ; l=0,1, \ldots,(p-1)\} .
$$

Effectively, the spectrum is obtained by starting with $p$ infinite sets of energy levels with equal spacing $\Omega_{p}$ and shifting these sets by successive arbitrary amounts $\omega_{0}, \omega_{1}, \ldots, \omega_{p-1}$. The superpotentials $W^{(p)}(x)$ corresponding to cyclic shape invariant potentials turn out to have rather interesting behavior at large $x$. One finds a linear dependence $\Omega_{p} x / 2 p$ corresponding to a harmonic oscillator of angular frequency $\Omega_{p} / p$ with superposed sinusoidal oscillations which become more closely spaced with increasing $x$. We find that these oscillations at large $x$ are given by the functional form $b_{p j} \sin \left(q_{p j} x^{2}\right)$. For any choice of the period $p$, there are several oscillation "frequencies" $q_{p j}$, with $j=1,2, \ldots,[p] / 2$, where $[p]$ is the largest even integer less than $p$. These "frequencies" are given by $q_{p j}=\Omega_{p} \tan (\pi j / p) / 2 p$. The amplitudes of the oscillations $b_{p j}$ depend on the energy spacings $\omega_{0}, \omega_{1}, \ldots$ The superpotentials also have unusual behavior at small $x$. For even values of $p$, one finds that $W^{(p)}(x)$ diverges like $1 / x$, whereas for odd values of $p, W^{(p)}(0)=0$. Our plan is to first review some basic ideas of supersymmetric quantum mechanics and the concept of shape invariance for a change of parameters $a_{1}=f\left(a_{0}\right)$. Next, we formulate the general set of coupled nonlinear differential equations governing cyclic shape invariant potentials of arbitrary period $p$. We describe some real transformations $f$ which have the property that $f^{p}\left(a_{0}\right)=a_{0}$, that is the parameters $a_{k}, k=0,1, \ldots,(p-1)$ repeat with period $p$. We study and discuss the solutions of the cyclic shape invariance conditions. The special cases of periods $p=3,4$ are treated in some detail. Many properties generalize to arbitrary values 
of $p$. Numerical solutions for $W^{(p)}(x)$ and the corresponding potentials $V_{-}^{(p)}(x)$ illustrate the general results. As an application, we examine cyclic shape invariant potentials corresponding to energy level spacings $\omega_{k}$ which are the periodic points generated by the logistic map $z_{k+1}=r z_{k}\left(1-z_{k}\right)$ [沟. As the parameter $r$ increases from 1 to 3.569946, the period $p$ also increases and the energy level spacings become increasingly more chaotic. This is reflected in the fact that the potentials $V_{-}(x)$ show increasingly more oscillation frequencies as one follows the period doubling route toward chaos.

\section{General Formalism for Cyclic Shape Invariant Potentials:}

Recall that the superpotential $W\left(x, a_{0}\right)$ generates the supersymmetric partner potentials

$$
V_{ \pm}\left(x, a_{0}\right)=W^{2}\left(x, a_{0}\right) \pm W^{\prime}\left(x, a_{0}\right)
$$

where $a_{0}$ is a set of parameters. These partner potentials are shape invariant if they both have the same $x$-dependence upto a change of parameters $a_{1}=f\left(a_{0}\right)$ and an additive constant $R\left(a_{0}\right)$. The shape invariance condition is [1] $V_{+}\left(x, a_{0}\right)=V_{-}\left(x, a_{1}\right)+R\left(a_{0}\right)$, or equivalently

$$
W^{2}\left(x, a_{0}\right)+W^{\prime}\left(x, a_{0}\right)=W^{2}\left(x, a_{1}\right)-W^{\prime}\left(x, a_{1}\right)+R\left(a_{0}\right)
$$

The property of shape invariance permits an immediate analytic determination of energy eigenvalues [1], eigenfunctions [5] and scattering matrices [6]. For unbroken supersymmetry, the eigenstates of the potential $V_{-}(x)$ are:

$$
\begin{gathered}
E_{0}^{(-)}=0, E_{n}^{(-)}=\sum_{k=0}^{n-1} R\left(a_{k}\right) \\
\psi_{0}^{(-)} \propto e^{-\int_{x_{0}}^{x} W\left(y, a_{0}\right) d y}, \psi_{n}^{(-)}\left(x, a_{0}\right)=\left[-\frac{d}{d x}+W\left(x, a_{0}\right)\right] \psi_{n-1}^{(-)}\left(x, a_{1}\right) \quad(n=1,2,3, \ldots) .
\end{gathered}
$$

The lowest lying eigenstate is at zero energy, characteristic of unbroken supersymmetry.

Solutions of the shape invariance condition (3) corresponding to a translational change of parameters $a_{1}=f\left(a_{0}\right)=a_{0}+\beta, a_{n}=f^{n}\left(a_{0}\right)=a_{0}+n \beta$ are well-known [3]. Also, the scaling change of parameters $a_{1}=f\left(a_{0}\right)=q a_{0}, a_{n}=f^{n}\left(a_{0}\right)=q^{n} a_{0}$ has been investigated and shown to produce new classes of shape invariant potentials [7, 8], which included selfsimilar potentials [9] as a special case. 
Very recently, another type of change of parameters defined by $f^{2}\left(a_{0}\right)=a_{0}$ was studied in detail [10]. Here, the parameters repeat after a cycle of two iterations and the spectrum consists of two shifted sets of equally spaced eigenvalues. In this paper, we consider a much broader change of parameters defined by

$$
f^{p}\left(a_{0}\right)=a_{0}
$$

where the parameters will repeat after a cycle of $p$ iterations. For this case, one has $a_{0}=$ $a_{p}, f\left(a_{0}\right)=a_{1}=a_{p+1}$, etc.

For a cyclic set of parameters, we now formulate the corresponding shape invariance conditions (3) for any period $p$. Defining $R\left(a_{k}\right) \equiv \omega_{k}$ for $k=0,1, \ldots,(p-1)$, and using $a_{p}=a_{0}$, one obtains a set of $p$ coupled nonlinear differential equations

$$
W^{2}\left(x, a_{k}\right)+W^{\prime}\left(x, a_{k}\right)=W^{2}\left(x, a_{k+1}\right)-W^{\prime}\left(x, a_{k+1}\right)+\omega_{k} \quad, \quad k=0,1, \ldots,(p-1) .
$$

There are $p$ unknown superpotentials $W\left(x, a_{k}\right)$ which are related to each other by cyclic permutations of the indices $0,1,2, \ldots,(p-1)$. We are seeking superpotentials with odd parity, which will yield symmetric potentials $V_{-}(x)$. It is convenient to denote $W\left(x, a_{k}\right) \equiv$ $W_{k}$. The set of shape invariance conditions now has a deceptively succinct form:

$$
W_{k}^{2}+W_{k}^{\prime}=W_{k+1}^{2}-W_{k+1}^{\prime}+\omega_{k} \quad, k=0,1, \ldots,(p-1) .
$$

There are two important problems one needs to solve: (i) to find real transformation functions $f(y)$ which satisfy eq. (5) and hence produce a cyclic set of parameters ; (ii) to determine the superpotentials $W_{k}^{(p)}(x)$ by solving the cyclic shape invariance conditions (7) corresponding to an arbitrary cyclic choice of parameters. These two problems will be treated in turn.

\section{Transformations Giving Cyclic Sets of Parameters:}

We now show that a simple algorithm for generating non-trivial real functions $f: \mathbf{R} \rightarrow \mathbf{R}$ satisfying $f^{p}(y)=y, \forall y \in \mathbf{R}$ is the projective(Moebius) transformation

$$
f(y)=\frac{a y+b}{c y+d}
$$

with specific constraints on the parameters $a, b, c, d$. Let $M$ be the real $2 \times 2$ matrix

$$
M=\left(\begin{array}{ll}
a & b \\
c & d
\end{array}\right) \text {. }
$$


For $f(y)$ given by eq. (8), observe that $f^{p}(y)$ is also a projective transformation whose coefficients are the same as the elements of the matrix $M^{p}$. Therefore, solving eq. (5) can be rephrased in terms of finding a real $2 \times 2$ matrix $M$, such that for any integer $p \geq 1$, the matrix $M^{p}$ is proportional to the unit matrix $\mathbf{1}$. Without loss of generality, we choose the condition $M^{p}=\mathbf{1}$, and our problem is to find real 2-dimensional representations of the cyclic group of order $p$. This group being Abelian, its 2-dimensional representations are reducible, i.e. there exists a non-singular matrix $Q$ for all matrices $M$ such that

$$
\mathcal{M} \equiv Q^{-1} M Q=\left(\begin{array}{cc}
\lambda_{1} & 0 \\
0 & \lambda_{2}
\end{array}\right) \text {. }
$$

Here $\lambda_{k}(k=1,2)$ stands for any two of the $p$-th roots of unity:

$$
\lambda_{k}=\exp \left(\frac{2 \pi j_{k}}{p} i\right), \quad j_{k}=0, \ldots, p-1 .
$$

Since we are interested in non-trivial matrices, we will impose two additional constraints: $\lambda_{1} \neq \lambda_{2}$ and $M^{n} \neq \mathbf{1}$ for any $n<p$. The range of possible solutions is constrained by the invariance under similarity transformations (10) of both the trace and the determinant:

$$
a+d=\exp \left(\frac{2 \pi j_{1}}{p} i\right)+\exp \left(\frac{2 \pi j_{2}}{p} i\right) ; a d-b c=\exp \left(\frac{2 \pi\left(j_{1}+j_{2}\right)}{p} i\right) .
$$

Also, the reality condition for the elements of $M$ gives

$$
\sin \left(\frac{2 \pi j_{1}}{p}\right)+\sin \left(\frac{2 \pi j_{2}}{p}\right)=0 ; \sin \left(\frac{2 \pi\left(j_{1}+j_{2}\right)}{p}\right)=0 .
$$

Taking into account all the above considerations we are left only with the roots of unity $\lambda_{1}$ and $\lambda_{2}$ satisfying $j_{1}+j_{2}=p$. Therefore, the matrix $\mathcal{M}$ will be a matrix of the form (10) with $\lambda_{1}=\exp \left(\frac{2 \pi j_{1}}{p} i\right)=\lambda_{2}^{-1}$. Note that the choice $j=0$ gives $\mathcal{M}=\mathbf{1}$, which gives a trivial $M$. Similarly, if $p$ is a non-prime number, say $p=j n$, then $\mathcal{M}^{n}=\mathbf{1}$ for $n<p$, which gives $M^{n}=\mathbf{1}$, and is not a non-trivial result for $M$. However, for all prime periods $p$, the matrix $M$ has the form (9) whose elements must be real and satisfy the system of equations:

$$
a d-b c=1 ; a+d=2 \cos \left(\frac{2 \pi j}{p}\right) \quad, \quad(j=0,1, \ldots, p-1) .
$$

Finally, the desired transformation function $f(y)$ is given by eq. (8) with real parameters $a, b, c$ and $d$ satisfying the constraints (14). For example, a projective transformation corresponding to the choices $p=3, j=1$ is

$$
f(y)=\frac{a y+b}{-y\left(a^{2}+a+1\right) / b-(a+1)},
$$


and it is easy to check that $f^{3}(y)=y$.

\section{Solutions of the Cyclic Shape Invariance Conditions:}

Having established that there exist real transformations $f$ which produce cyclic sets of parameters, we now turn our attention to studying the solutions of the cyclic set of equations (17). A very general result comes from adding all the equations. It gives $\sum_{k=0}^{p-1} W_{k}^{\prime}=\Omega_{p} / 2$, which on integration yields

$$
\sum_{k=0}^{p-1} W_{k}(x)=\frac{1}{2} \Omega_{p} x .
$$

This condition is valid for all $x$. There is no constant of integration in eq. (15) since we want the superpotentials $W_{k}$ to be odd functions of $x$. In particular, at large $x$, constraints (7) and (15) give all $W_{k}$ the linear asymptotic behaviour $\Omega_{p} x / 2 p$, corresponding to a simple harmonic oscillator potential of angular frequency $\Omega_{p} / p$. Also, at small $x$, in order to satisfy eqs. (7), all superpotentials can at most have a $1 / x$ singularity.

The general analytic solution of equations (]) is not easy to obtain. However, a numerical solution can be obtained via the Runge-Kutta method and we will also obtain several analytic results at small and large values of $x$. For ease of presentation, we systematically consider small values of the cycle period $p$ before coming to general results.

$p=1: \quad$ Here $a_{1}=f\left(a_{0}\right)=a_{0}$ and all energy levels are equally spaced. As expected, the shape invariance condition (7) gives $W_{0}^{(1)}=\omega_{0} x / 2$ which is a simple harmonic oscillator superpotential.

$p=2: \quad$ This is the case of two shifted sets of equally spaced eigenvalues considered in detail in ref. [10]. The shape invariance conditions (7) are:

$$
W_{0}^{2}+W_{0}^{\prime}=W_{1}^{2}-W_{1}^{\prime}+\omega_{0} \quad, \quad W_{1}^{2}+W_{1}^{\prime}=W_{0}^{2}-W_{0}^{\prime}+\omega_{1}
$$

The solution is obtained by straightforward manipulations. The superpotential $W_{0}^{(2)} \equiv$ $W^{(2)}\left(x, a_{0}\right)$ and the corresponding potential $V_{-}^{(2)}(x)$ obtained using eq. (2) are given by

$$
W_{0}^{(2)}(x)=\frac{\Omega_{2} x}{4}+\frac{\left(\omega_{0}-\omega_{1}\right)}{2 \Omega_{2} x}, V_{-}^{(2)}(x)=\frac{\left(\omega_{0}-\omega_{1}\right)\left(3 \omega_{0}+\omega_{1}\right)}{4 \Omega_{2}^{2} x^{2}}-\frac{\omega_{1}}{2}+\frac{\Omega_{2}^{2} x^{2}}{16} .
$$

Note that for period $p=2$, the superpotential $W_{0}^{(2)}$ diverges like $\alpha_{0} / x$ near the origin, which produces a singular inverse square potential in the transition region [11, 12, 13]. A 
detailed discussion of the potential as well as the eigenfunctions $\psi_{n}^{(-)}$obtained from eq. (4) is given in ref. [10].

$p=3: \quad$ There are now three shape invariance conditions (元):

$$
W_{0}^{2}+W_{0}^{\prime}=W_{1}^{2}-W_{1}^{\prime}+\omega_{0}, W_{1}^{2}+W_{1}^{\prime}=W_{2}^{2}-W_{2}^{\prime}+\omega_{1}, W_{2}^{2}+W_{2}^{\prime}=W_{0}^{2}-W_{0}^{\prime}+\omega_{2} .
$$

These equations are not easy to solve analytically. In order to obtain a numerical solution, it is best to first solve eqs. (18) for the derivatives $W_{0}^{\prime}, W_{1}^{\prime}, W_{2}^{\prime}$. One gets

$$
\begin{aligned}
& W_{0}^{\prime}=W_{1}^{2}-W_{2}^{2}+\frac{1}{2}\left(\omega_{0}-\omega_{1}+\omega_{2}\right), \\
& W_{1}^{\prime}=W_{2}^{2}-W_{0}^{2}+\frac{1}{2}\left(\omega_{1}-\omega_{2}+\omega_{0}\right), \\
& W_{2}^{\prime}=W_{0}^{2}-W_{1}^{2}+\frac{1}{2}\left(\omega_{2}-\omega_{0}+\omega_{1}\right) .
\end{aligned}
$$

In this form the Runge-Kutta method is immediately applicable, and the superpotentials can be determined for any choice of the energy spacings $\omega_{0}, \omega_{1}, \omega_{2}$. An illustration of the results is shown in Fig. 2. The overall average behavior of $W_{0}^{(3)}$ at large $x$ is indeed seen to be $\Omega_{3} x / 6$ as expected. On this linear dependence are clear oscillations which get more closely spaced as $x$ increases. They correspond to a behavior $b \sin \left(q x^{2}\right)$ where the amplitude $b$ and the frequency $q$ both depend on the choice of energy spacings $\omega_{0}, \omega_{1}, \omega_{2}$. In particular, when one chooses $\omega_{0}=\omega_{1}=\omega_{2}$, the numerical solution shows no oscillations. This is reasonable since for this case one expects a simple harmonic superpotential $\Omega_{3} x / 6$.

One can get a number of analytic results for eqs. (19) at small $x$ as well as large $x$. At small $x$, if one feeds the series expansions

$$
W_{k}=\alpha_{k} / x+\beta_{k} x+\gamma_{k} x^{3}+\delta_{k} x^{5}+\cdots
$$

for $k=0,1,2$ into eq. (19) and equates coefficients of $x$, one gets

$$
W_{0}^{(3)}=\frac{x}{2}\left(\omega_{0}-\omega_{1}+\omega_{2}\right)+\frac{x^{3}}{3} \omega_{1}\left(\omega_{0}-\omega_{2}\right)+\frac{x^{5}}{15} \omega_{1}\left(\omega_{0} \omega_{1}+\omega_{1} \omega_{2}-\omega_{0}^{2}-\omega_{2}^{2}\right)+\cdots
$$

with $W_{1}^{(3)}, W_{2}^{(3)}$ obtained by cyclic permutations of the indices $0,1,2 . W_{0}^{(3)}$ vanishes at $x=0$. Note that $\sum_{k=0}^{2} \gamma_{k}=\sum_{k=0}^{2} \delta_{k}=0$ in agreement with eq. (15). At large $x$, one finds oscillations with one frequency $q_{31}=\Omega_{3} \sqrt{3} / 6$. This is a special case of the result for arbitrary $p$ which will be derived below. 
$p=4: \quad$ It is worth discussing period 4 briefly, since several results are different from period 3. There are four cyclic shape invariance conditions:

$$
\begin{aligned}
& W_{0}^{2}+W_{0}^{\prime}=W_{1}^{2}-W_{1}^{\prime}+\omega_{0}, W_{1}^{2}+W_{1}^{\prime}=W_{2}^{2}-W_{2}^{\prime}+\omega_{1}, \\
& W_{2}^{2}+W_{2}^{\prime}=W_{3}^{2}-W_{3}^{\prime}+\omega_{2}, W_{3}^{2}+W_{3}^{\prime}=W_{0}^{2}-W_{0}^{\prime}+\omega_{3} .
\end{aligned}
$$

Unlike the period 3 case, one cannot directly solve for $W_{0}^{\prime}, W_{1}^{\prime}, W_{2}^{\prime}, W_{3}^{\prime}$, since their coefficients in eqs. (22) have a zero determinant. Indeed, this situation occurs for all even periods $p$. For the case of period 4, one can proceed by noting that eqs. (22) give the constraint $W_{0}^{2}-W_{1}^{2}+W_{2}^{2}-W_{3}^{2}=\left(\omega_{0}-\omega_{1}+\omega_{2}-\omega_{3}\right) / 2$, which on differentiation yields

$$
W_{0} W_{0}^{\prime}-W_{1} W_{1}^{\prime}+W_{2} W_{2}^{\prime}-W_{3} W_{3}^{\prime}=0
$$

This equation, along with any three of eqs. (22), permits one to solve for $W_{0}^{\prime}, W_{1}^{\prime}, W_{2}^{\prime}, W_{3}^{\prime}$, and proceed with a numerical determination of the superpotentials by the Runge-Kutta method.

Analytically, at small $x$, substitution of expansions of the form (20) into eqs. (22) and collecting powers of $x$ gives

$$
W_{0}^{(4)}=\frac{\left(\omega_{0}-\omega_{1}+\omega_{2}-\omega_{3}\right)}{2 \Omega_{4} x}+\frac{x \Omega_{4}\left(\omega_{0} \omega_{3}+\omega_{0} \omega_{1}+\omega_{2} \omega_{3}-\omega_{2} \omega_{1}\right)}{4\left(\omega_{0}+\omega_{2}\right)\left(\omega_{1}+\omega_{3}\right)}+\cdots
$$

with the other superpotentials $W_{1}^{(4)}, W_{2}^{(4)}, W_{3}^{(4)}$ obtained by cyclic permutations of the indices $0,1,2,3$. Note the singular $1 / x$ dependence, similar to period 2 . This is a feature of all even periods. At large $x$, period 4 superpotentials show oscillations with a single frequency $q_{41}=\Omega_{4} / 8$. We will derive the general result for arbitrary $p$ below.

Results for Arbitrary $p$ : The experience gained from small values of the period $p$ can be used to derive a number of general analytic statements valid for any arbitrary period $p$. At small $x$, the superpotential $W_{0}^{(p)}(x)$ has the form

$$
\begin{aligned}
W_{0}^{(p)} & =\frac{x}{2}\left(\omega_{0}-\omega_{1}+\cdots+\omega_{p-1}\right) \\
& -\frac{x^{3}}{3}\left[\omega_{1}\left(\omega_{2}-\omega_{3}+\cdots+\omega_{p-1}-\omega_{0}\right)+\omega_{3}\left(\omega_{4}-\omega_{5}+\cdots+\omega_{p-1}-\omega_{0}+\omega_{1}-\omega_{2}\right)\right. \\
& \left.+\cdots+\omega_{p-2}\left(\omega_{p-1}-\omega_{0}+\omega_{1}-\cdots-\omega_{p-3}\right)\right]+O\left(x^{5}\right) ; \quad(p \text { odd }) \\
W_{0}^{(p)} & =\frac{1}{2 \Omega_{p} x}\left(\omega_{0}-\omega_{1}+\omega_{2}-\cdots-\omega_{p-1}\right)+\frac{x \Omega_{p}}{4}[1-2 \times
\end{aligned}
$$




$$
\begin{aligned}
& \left.\times \frac{\omega_{1}\left(\omega_{2}+\omega_{4}+\cdots+\omega_{p-2}\right)+\omega_{3}\left(\omega_{4}+\omega_{6}+\cdots+\omega_{p-2}\right)+\cdots+\omega_{p-3} \omega_{p-2}}{\left(\omega_{0}+\omega_{2}+\cdots+\omega_{p-2}\right)\left(\omega_{1}+\omega_{3}+\cdots+\omega_{p-1}\right)}\right] \\
& +O\left(x^{3}\right) ; \quad(p \text { even })
\end{aligned}
$$

The behaviour of the other superpotentials $W_{k}^{(p)}(x)$ can be seen by cyclically permuting the indices $0,1,2, \ldots,(p-1)$. Note the $1 / x$ singularity at the origin for even periods. As for the case of periods $p=2,4$, it can be readily verified that the coefficient of the $1 / x^{2}$ singularity in the potential $V_{k}^{(p)}$ lies in the transition region $-1 / 4$ to $3 / 4$. In this region, both solutions of the Schrödinger equation are square integrable at the origin [11, 12, 13]. The infinite discontinuity in $W^{(p)}(x)$ at the origin for even $p$ produces a $\delta(x)$ term in the potential $V_{-}^{(p)}(x)$ as described in ref. ([10]).

At large $x$, one removes the asymptotic linear behaviour by defining

$$
Q_{k}(x) \equiv W_{k}(x)-\Omega_{p} x / 2 p, \quad k=0,1, \ldots, p-1
$$

Since numerical solutions suggest sinusoidal oscillations in $x^{2}$, we make a change of variables to $u \equiv x^{2}$. In terms of $u$, the equations satisfied by $Q_{k}$ are obtained by substituting eq. (25) into (7). Retaining only the leading powers of $u$ yields

$$
Q_{0}^{\prime}+Q_{1}^{\prime}=\frac{\Omega_{p}}{2 p}\left(-Q_{0}+Q_{1}\right)
$$

and cyclic permutations. This set of equations can be written in a more compact form by putting all the superpotentials into a column vector $Q$ and making use of the matrix $C_{p}$ whose only non-zero elements are $\left(C_{p}\right)_{12}=\left(C_{p}\right)_{23}=\cdots=\left(C_{p}\right)_{p-1, p}=\left(C_{p}\right)_{p 1}=1$. The equations now read

$$
\left(C_{p}+1\right) Q^{\prime}-\frac{\Omega_{p}}{2 p}\left(C_{p}-1\right) Q=0
$$

The most general solution is a linear combination of eigenstates of the form $Q \propto \exp (\lambda u)$. The eigenvalues $\lambda$ are determined from

$$
\operatorname{det}\left[\lambda\left(C_{p}+1\right)-\left(\Omega_{p} / 2 p\right)\left(C_{p}-1\right)\right]=0
$$

Defining $\tilde{\lambda}=2 p \lambda / \Omega_{p}$, and expanding the determinant, eq. (28) can be re-written as

$$
(\tilde{\lambda}+1)^{p}-(-1)^{p}(\tilde{\lambda}-1)^{p}=0
$$

which is a polynomial equation of degree $p$ for $p$ odd and of degree $p-1$ for $p$ even. This situation occurs because eq. (28) is more general than the standard eigenvalue equation. 
The solution is

$$
\frac{1-\tilde{\lambda}}{1+\tilde{\lambda}}=\exp \left(\frac{2 \pi j}{p} i\right), \quad j=0, \pm 1, \pm 2, \cdots, \pm[p] / 2
$$

$[p]$ is the largest even integer less than $p$. This gives rise to distinct oscillation frequencies

$$
q_{p j}=\left(\Omega_{p} / 2 p\right) \tan (\pi j / p), j=1,2, \ldots,[p] / 2
$$

At large $x$, the superpotential $W_{0}^{(p)}(x)$ has the form

$$
W_{k}^{(p)}(x)=\frac{\Omega_{p} x}{2 p}+\sum_{j} b_{p j} \sin \left(q_{p j} x^{2}+\phi_{j}^{(k)}\right)+O(1 / x)
$$

where the phases are

$$
\phi_{j}^{(k)}=\phi_{j}+2 \pi k / p
$$

Note that the requirement of having odd superpotentials can be satisfied by inserting an asymptotically constant odd function such as $\tanh (\alpha x)$ or $\tan ^{-1}(\alpha x)$ with the coefficient $b_{p j}$. This does not affect the above discussion of oscillation frequencies. Also, if one uses eq. (四) to get the asymptotic behaviour of wave functions, the linear term in $W^{(p)}$ gives the familiar Gaussian dependence of a harmonic oscillator and the sinusoidal correction terms in $W^{(p)}$ give Fresnel integrals producing oscillations on the Gaussian.

As an example, for period 5 , taking $\Omega_{5}=6.5$, we predict two oscillation frequencies $q_{51}=0.472$ and $q_{52}=2.001$ from eq. (29), and we have checked that these values are in agreement with the numerical solutions shown in Fig. 3.

\section{Approach to Quantum Chaos Using the Logistic Map:}

We have now established the method of generating cyclic shape invariant potentials. This method can be applied using any transformation $f(y)$ which generates parameters which repeat with period $p$. For the special projective transformations described previously, the initial parameter $a_{0}$ can be chosen arbitrarily. There are also other possible transformations in which cyclic parameters are obtained if one starts from specific choices

for $a_{0}$. One such interesting possibility, which we discuss in detail here, is to use a set of cyclic parameters given by the periodic points of the logistic map

$$
z_{k+1}=r z_{k}\left(1-z_{k}\right)
$$

This famous transformation has been extensively studied 㚪. For $1<r<3$, one has a single non-zero fixed point at $z=(r-1) / r$; for $3<r<3.449499$, there is a stable 2-cycle 
with periodic points at $z=[(r+1) \pm \sqrt{(r-3)(r+1)}] / 2 r$; for $3.449499<r<3.544090$, there is a stable 4-cycle, etc. As $r$ is increased, the period keeps doubling, giving finally the period $2^{\infty}$ at 3.569946. For $r>3.569946$, one obtains all odd periods and their harmonics, and at $r>4$, there is complete chaos.

In our application, we will identify the energy spacings $\omega_{k}$ with the periodic points of the logistic map. For example, if we choose $r=3.20$, the periodic points are 0.799 and 0.513 , and we determine the potentials which have energy spacings $\omega_{0}=0.799$ and $\omega_{1}=0.513$. The corresponding superpotential is shown in Fig 4(a). Other examples with larger values of $r$ and correspondingly larger periods $p=4,8$ are also shown in Figs. 4(b) and 4(c). Clearly, as one advances along the period doubling route to chaos, more and more oscillation frequencies come into play - which is certainly a plausible result. Specifically, for period $2^{n}$, there are $2^{n-1}-1$ frequencies given by eq. (29).

Although we have so far chosen values of $r$ which yield stable cycles of period $2^{n}$, it is known that there are small windows of $r$-values where stable cycles of periods $3,5, \ldots$ also exist. The above methods immediately permit us to obtain superpotentials for these cases also. These issues and deeper connections with chaotically spaced energy levels will be studied further. Parenthetically, we note that the locations of the nontrivial zeros of the Riemann zeta function appear to exhibit chaotic behaviour. If these positions are identified with energy level spacings, the corresponding potentials have many oscillations [14.

We would like to thank A. Gangopadhyaya and T. Imbo for valuable discussions and comments. U.S. acknowledges the hospitality of the Institute of Physics, Bhubaneswar where part of this work was done. This research was supported in part by the U.S. Department of Energy. 


\section{Figure Captions}

Figure 1: This diagram illustrates the energy levels of a cyclic shape invariant potential of period $p$. The lowest level is at zero energy, and the other excited energy levels are given by (曾).

Figure 2: The superpotential $W_{0}^{(3)}(x)$ and the corresponding symmetric potential $V_{-}^{(3)}(x)$ for period $p=3$ and a choice of energy level spacings $\omega_{0}=1.0, \omega_{1}=0.5, \omega_{2}=2.3$. The superpotential was obtained by a numerical solution of eqs. (19) by the Runge-Kutta method. The oscillation frequency given by eq. (29) is $q_{31}=1.097$, and is in agreement with the figure. This is established by counting the number of maxima in any chosen interval of $x$ values. The energy levels for this potential are given by expression (11) and are located at $n \omega_{3}, n \omega_{3}+1.0, n \omega_{3}+1.5$ with $\omega_{3}=3.8$ and $n=0,1,2, \ldots$

Figure 3: A plot of the superpotential $W_{0}^{(5)}-\frac{\Omega_{5} x}{10}$ for period $p=5$ and energy level spacings $\omega_{0}=0.8, \omega_{1}=1.5, \omega_{2}=1.0, \omega_{3}=3.0, \omega_{4}=0.2$. The asymptotic behavior $\frac{\Omega_{5} x}{10}$ has been subtracted in order to clearly reveal the two oscillation frequencies $q_{51}=0.4722$ and $q_{52}=2.001$, predicted by eq. (29). The maxima are spaced in agreement with the smaller frequency $q_{51}$ whereas their "envelope" corresponds to the larger frequency $q_{52}$.

Figure 4: This figure shows how the superpotential $W_{0}^{(p)}(x)-\frac{\Omega_{p} x}{2 p}$ gets progressively more complicated and aquires more oscillation frequencies as the period $p$ is successively doubled along the bifurcation route to chaos via the logistic map (32). The parameter values chosen for the figures are:

(a) $r=3.200$ which gives period $p=2$ and a stable 2-cycle with energy spacings $\omega_{0}=0.799, \omega_{1}=0.513$.

(b) $r=3.500$ which gives period $p=4$ and a stable 4-cycle with $\omega_{0}=0.827, \omega_{1}=$ 0.501, $\omega_{2}=0.875, \omega_{3}=0.383$. The oscillation frequency from eq. (29) is $q_{41}=0.323$.

(c) $r=3.561$ which gives period $p=8$ and a stable 8-cycle with $\omega_{0}=0.890, \omega_{1}=$ $0.348, \omega_{2}=0.808, \omega_{3}=0.552, \omega_{4}=0.881, \omega_{5}=0.374, \omega_{6}=0.834, \omega_{7}=0.494$. The three oscillation frequencies given by eq. (29) are $q_{81}=0.1341, q_{82}=0.3238, q_{83}=0.7818$. 


\section{References}

[1] L. Gendenshtein, JETP Letters 38 (1983) 356.

[2] E. Witten, Nucl. Phys. B188 (1981) 513.

[3] For a recent review of supersymmetric quantum mechanics and additional references see F. Cooper, A. Khare and U. Sukhatme, Phys. Rep. 251 (1995) 267.

[4] For a description of the logistic map, see for example, H. Peitgen, H. Jürgens and D. Saupe, Fractals, Springer-Verlag (1992).

[5] R. Dutt, A. Khare and U. Sukhatme, Phys. Lett. 181B (1986) 295; J. Dabrowska, A. Khare and U. Sukhatme, Jour. Phys. A21 (1988) L195.

[6] A. Khare and U. Sukhatme, Jour. Phys. A21 (1988) L501.

[7] A. Khare and U. Sukhatme, Jour. Phys. A26 (1993) L901.

[8] D. Barclay, R. Dutt, A. Gangopadhyaya, A. Khare, A. Pagnamenta and U. Sukhatme, Phys. Rev. A48 (1993) 2786.

[9] A. Shabat, Inverse Prob. 8 (1992) 303; V. Spiridonov, Phys. Rev. Lett. 69 (1992) 398.

[10] A. Gangopadhyaya and U. Sukhatme, Phys. Lett. A224 (1996) 5.

[11] L. D. Landau and E. M. Lifshitz, Quantum Mechanics, Pergamon Press (1977).

[12] W. M. Frank, D. J. Land and R. M. Spector, Rev. Mod. Phys. 43 (1971) 36.

[13] A. Gangopadhyaya, P. K. Panigrahi and U. P. Sukhatme, J. Phys. A27 (1994) 4295.

[14] H. Wu and D. Sprung, Phys. Rev. E48 (1993) 2595. 


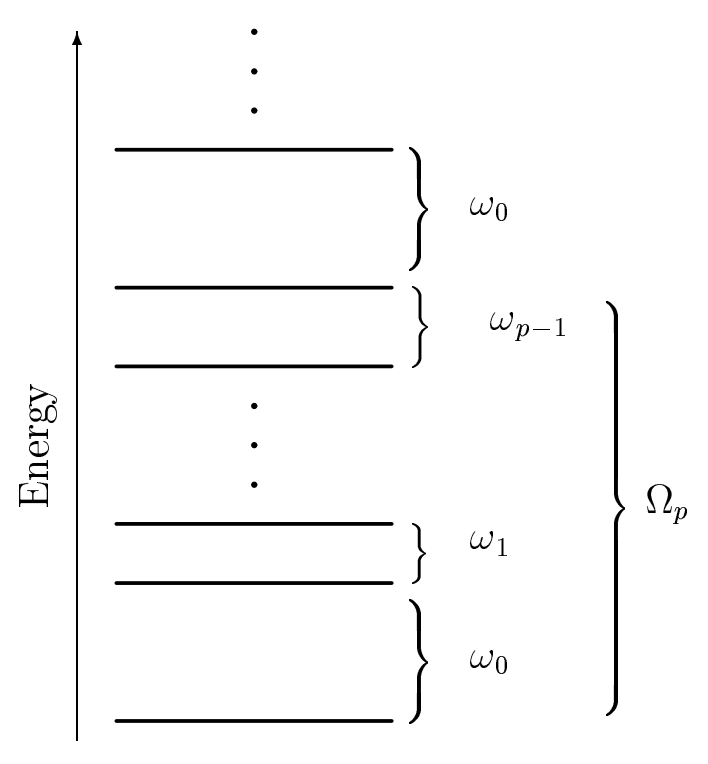

Figure 1: 

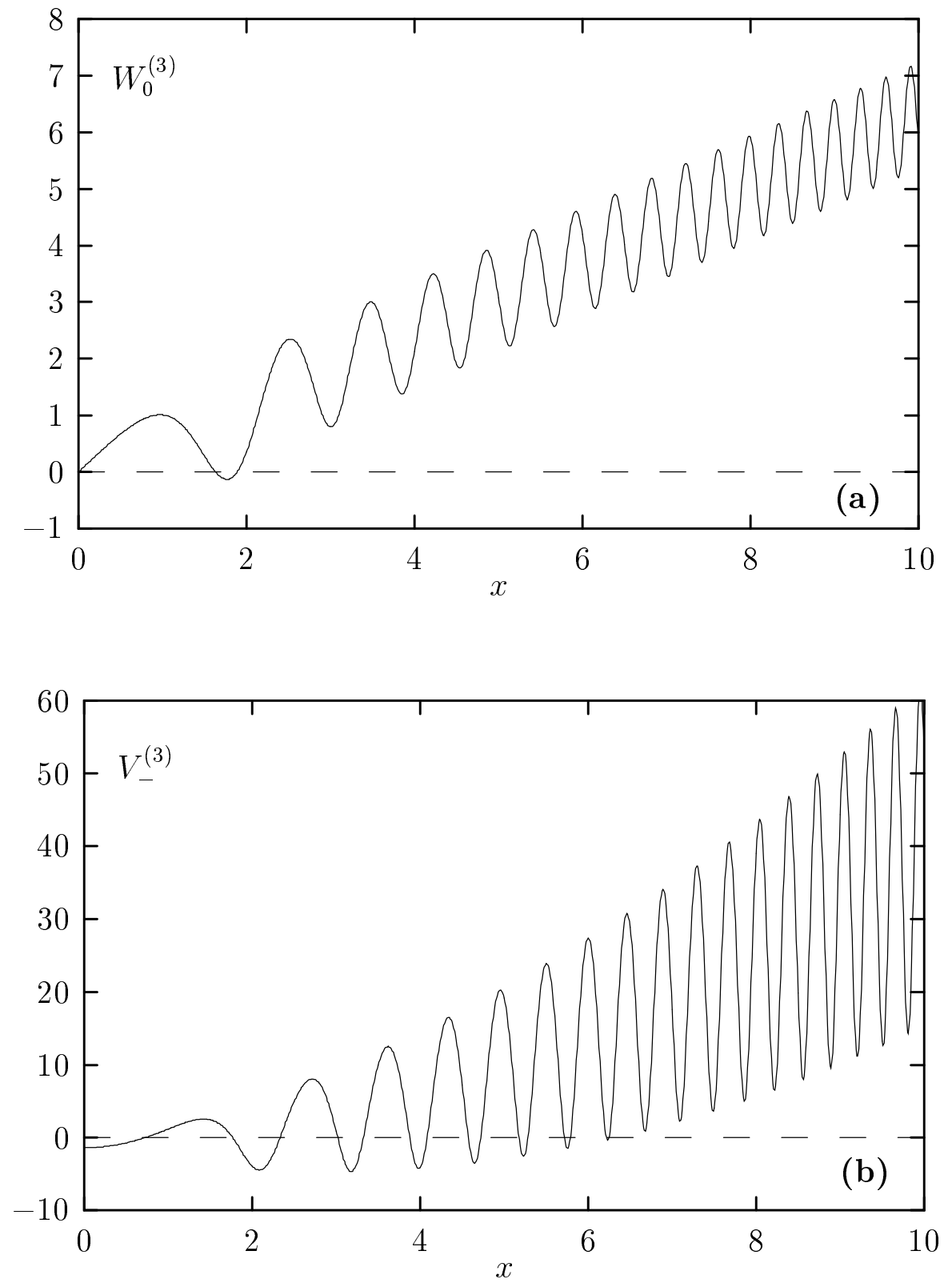

Figure 2: 


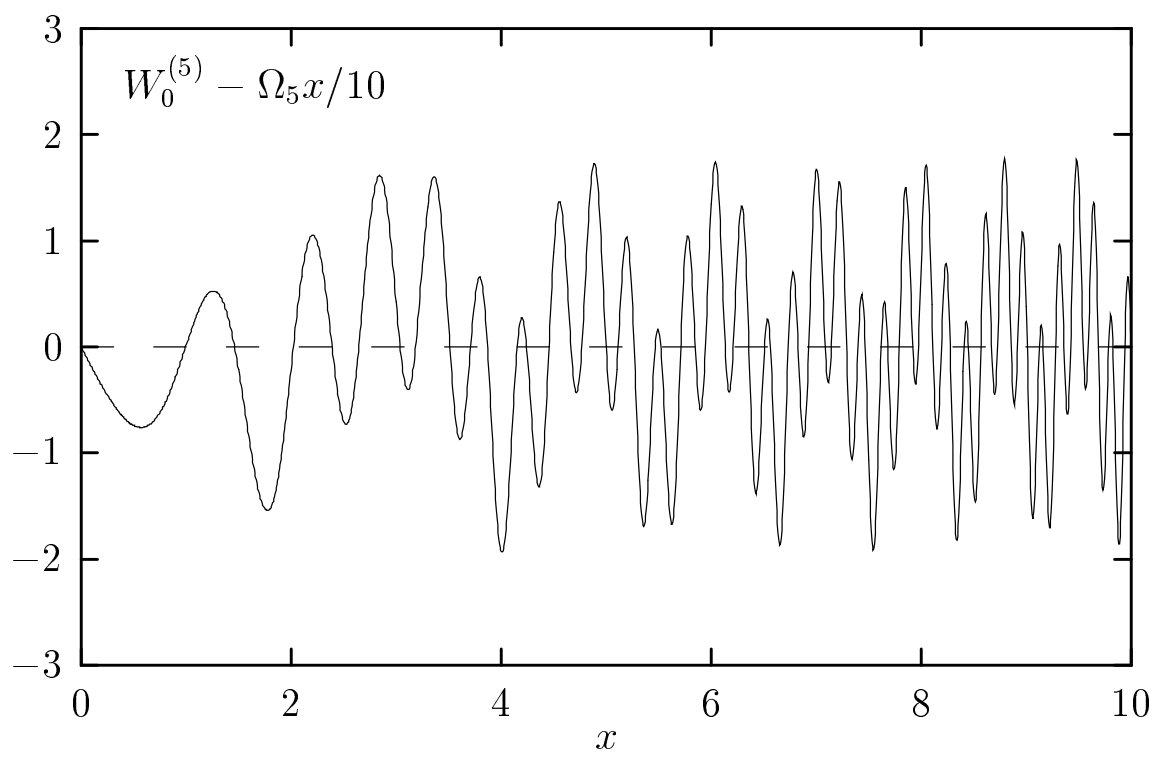

Figure 3: 

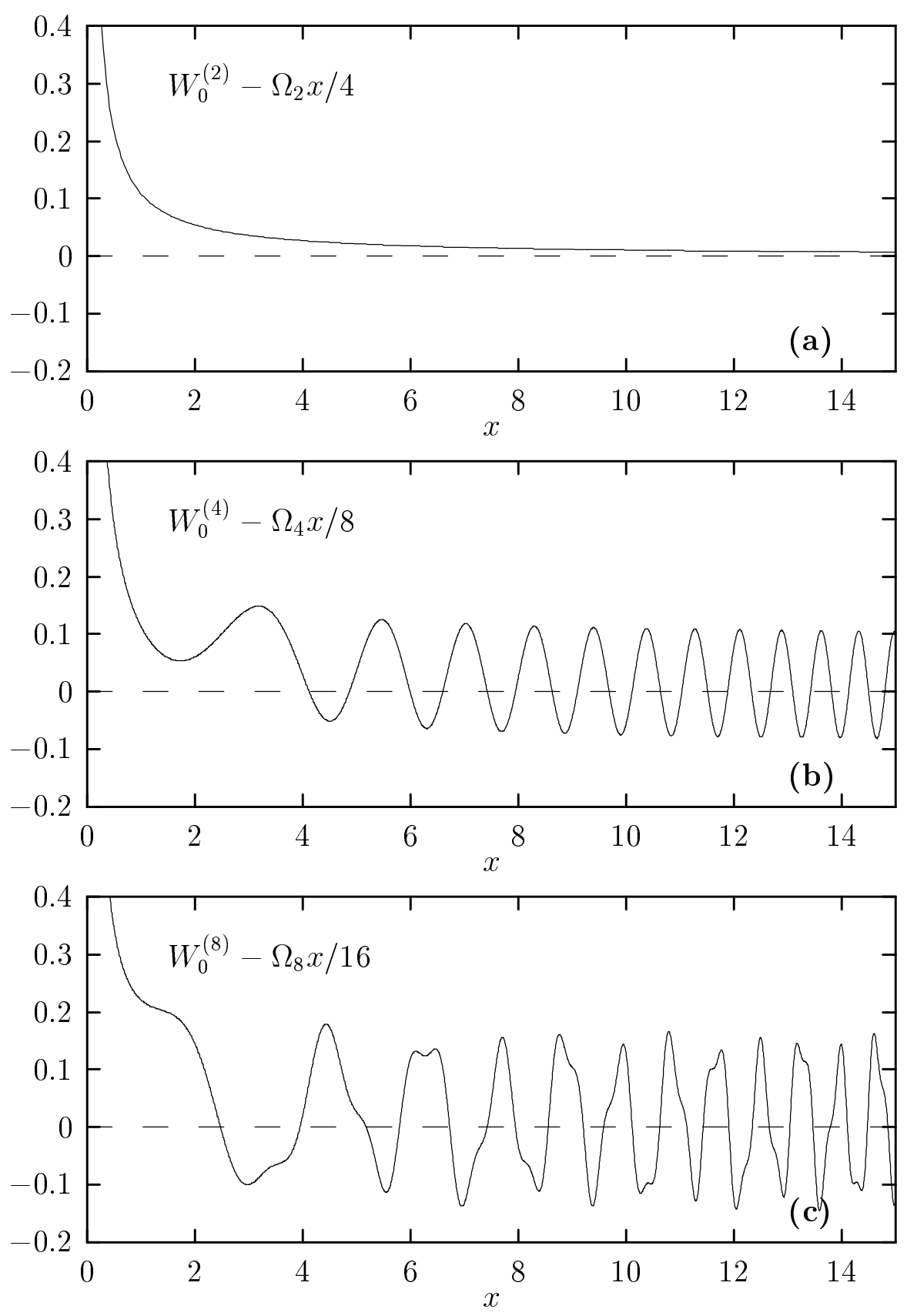

Figure 4: 